\section{Cahiers de Narratologie}

Analyse et théorie narratives

$10.2 \mid 2001$

La voix narrative

\title{
Phraséologie « soft » et voix postmoderne
}

\section{Christian Boix}

\section{OpenEdition}

Journals

Édition électronique

URL : http://journals.openedition.org/narratologie/10167

DOI : 10.4000/narratologie.10167

ISSN : 1765-307X

Éditeur

LIRCES

Édition imprimée

Date de publication : 1 janvier 2001

Pagination : 7-20

ISBN : 2914561032

ISSN : 0993-8516

Référence électronique

Christian Boix, «Phraséologie « soft » et voix postmoderne », Cahiers de Narratologie [En ligne], 10.2 | 2001, mis en ligne le 01 janvier 2001, consulté le 11 juin 2020. URL : http://journals.openedition.org/ narratologie/10167 ; DOI : https://doi.org/10.4000/narratologie.10167 


\title{
PHRASÉOLOGIE «SOFT » ET VOIX POSTMODERNE
}

\author{
Christian BOIX \\ Université de Bourgogne
}

L'approche de la Voix sera entendue ici sous un jour quelque peu différent de ce que recouvre ce concept au sein de la Narratologie "pure et dure ", de Todorov à Genette par exemple. J'ai déjà proposé, dans le cadre des travaux du CNA de Nicel, de sortir progressivement d'un ordre structural aux frontières franches - pouvant être repérées sur la base de phénomènes strictement morphologiques (simples, récurrents et invariables) - pour entrer dans l'ordre dynamique de la stratégie complexe. Disons, pour me résumer très sommairement, que la Voix représente un phénomène d'émergence, l'émergence d'une sorte de corporéité figurative qui peut se donner à voir sous n'importe quel type de discours, fictionnel ou non fictionnel, littéraire ou non littéraire, pourvu d'un référent réel ou imaginaire. On pourrait rapprocher cette vision de la Voix de ce que je nommerai la Loquence, ensemble organisé de gestes langagiers qui se constitue en signalement d'un locuteur (ce dernier pouvant incarner des tendances collectives d'un lieu ou d'une époque donnés).

C'est dans ce cadre que s'inscrit cette réflexion sur la phraséologie «soft » et la voix postmoderne. Une tendance forte se fait jour depuis les toutes dernières décennies, vraisemblablement inspirée par la généralisation d'une attitude

1 Cf. C. BOIX, "Les outils d'analyse de la voix narrative ", in Cahiers de Narratologie $\mathrm{n}^{\circ} 9$, Espace et voix narrative, CNA, Publications de la Faculté des Lettres, Arts et Sciences Humaines, Université de Nice-Sophia Antipolis, 1999, p. 159-173. Pour un exemple d'application, cf. C. BOIX : « La voix des personnages. Pepe Carvalho dans Los Mares del Sur ", in Cahiers de Narratologie n ${ }^{\circ} 6$, Le personnage romanesque, CNA, Publications de la Faculté des Lettres, Arts et Sciences Humaines, Université de Nice-Sophia Antipolis, 1994, p. 155-162. 
langagière "politically correct». Cette lame de fond semble toucher à tous les domaines, du politique à la publicité et à la communication, avec le dogme du «mieux-disant», en passant par une détension qui affecte aussi bien des mouvements littéraires que la structure argumentative des éditoriaux journalistiques, ou le langage ordinaire de la presse et des institutions au sein duquel il est fait grand usage de la figure rhétorique de l'euphémisme. Partout, à l'instar des yaourts et des sauces, le langage prend des airs allégés, la voix s'adoucit pour rejoindre le modèle souriant que l'on inculque aux hôtesses d'accueil téléphonique. Cette émergence postmoderne, cette corporéité particulière de la voix, ce soubassement que constitue la Loquence, relève du mode profond du sentir, antérieur à celui de la connaissance raisonnée. Sans doute est-ce dans ce «terreau » que poussent les racines de modes nouveaux d'appréhension de la réalité. Il est encore un peu tôt pour mesurer toutes les implications de ce mécanisme: je me contenterai donc de prendre quelques exemples d'actualisation discursive de cette tendance.

\section{DÉTENSION SÉMIOTIQUE ET LITTÉRATURE De l'engagement polémique à l'éthique conversationnelle}

L'un des symptômes de la mutation contemporaine de la Voix peut être trouvé dans ce que les sémioticiens nomment le TENSIF. Le premier ouvrage publié sur cette question avait pour titre : "Sémiotique des passions. Des états de choses aux états d'âme $»^{2}$. Les auteurs cherchaient à mettre en évidence, dans l'investissement discursif, un univers en deçà du sujet de l'énonciation: un univers plus élémentaire, pré-cognitif, antérieur à la conscience claire du "connaître ", le tensif relevant d'une catégorie où se manifeste la "sensibilité à ». Ainsi, toute configuration discursive serait parcourue par un "style sémiotique " particulier: état d'âme inquiet ou déprimé, tendu ou détendu, fébrile ou calme, impulsif ou

2 A.-J GReimas, J. FonTANILle, Sémiotique des passions. Des états de choses aux états d'âme, Paris, Seuil, 1991. 
lymphatique ${ }^{3}$. Ces recherches permettent de compléter la vision discontinuiste du langage par une dimension organique et modulée, et elles sont d'un grand intérêt pour faire apparaître des « formants » de la Voix discursive.

Parallèlement à cette perspective théorique, la dé-tension se donne à lire intuitivement dans la Voix de nombre d'auteurs littéraires. Prenons par exemple la mue de bon nombre d'auteurs espagnols lors du tournant de la Transition Démocratique. Un poète comme Gil de Biedma, engagé dans le combat idéologique anti-franquiste, avait merveilleusement su traduire ${ }^{4}$ la tension d'un auditoire buvant littéralement les paroles de la philosophe María Zambrano, la communion avec les idées qu'elle défendait :

Aquí en la Plaza del Pueblo

Se oía latir - y yo,

junto a ese balcón abierto,

era también un latido, escuchando.
Ici, sur la Place du Peuple

On entendait battre les cœurs

- et moi, tout contre de ce balcon ouvert, j'étais aussi un battement de cœur, écoutant.

Cette tonalité discursive fait d'autant mieux ressortir le contraste avec ce que le poète écrit quelques années plus tard, sur la quatrième de couverture de l'édition Seix Barral où il réunit ses poèmes :

Me quedé calvo en 1962 ; la pérdida me fastidia pero no me obsesiona - dicen que tengo una línea de cabeza muy buena. Gano bastante dinero. No ahorro. He sido de izquierda y es muy probable que siga siéndolo, pero hace tiempo que no ejerzo 5 .

Pour ironique que soit le propos, la Voix a changé, la tension également, dans le sens de la distance, du relâchement

3 Pour une première expérience de mise en évidence de ces phénomènes discursifs inarticulés, on se reportera à l'ouvrage d'Anne HÉNAUT : Le pouvoir comme passion, Paris, PUF, 1994.

4 J. GIL DE BIEDMA, Las personas del verbo, Barcelona, Seix Barral, Biblioteca Breve, 1991, p. 70.

5 Ibid. (quatrième de couverture). "Je suis devenu chauve en 1962 ; cette perte m'ennuie mais je n'en fais pas une obsession - on dit que le profil de mon crâne est excellent. Je gagne plutôt bien ma vie. Je ne fais pas d'économies. J'ai été de gauche et il est très probable que je le sois encore, mais il y a un certain temps que je n'exerce plus. " 
tensif qui convoque un destinataire avec qui on partage une connivence. La volonté de frapper l'esprit et de convaincre est remplacée par le ton du clin d'œil, de la plaisanterie amicale. Antérieurement engagé dans un combat, dans la défense de valeurs morales et humaines différentes de celles qui régissaient la société du Général Franco, l'intellectuel se retrouve quelques années plus tard dans un monde qui n'est pas forcément le Paradis qu'il attendait... Mais il parle dorénavant sur un ton dépassionné.

Cette volte-face vocale et passionnelle a été magistralement mise en scène par Eduardo MENDOZA dans son roman La verdad sobre el caso Savolta [La vérité sur l'affaire Savolta]. Publié l'année même de la mort de Franco, salué par tous comme une œuvre-charnière emblématique de la Transition, ce roman énonce très clairement le changement chronologique entre deux états tensifs du discours des protagonistes, le premier marqué comme ancien et le second comme nouveau :

Tampoco nuestras charlas derivaron en apasionadas polémicas, como había sucedido con Pajarito de Soto a poco de conocernos: esas acaloradas discusiones que ahora, en el recuerdo, acrecientan su importancia y se convierten en el símbolo nostálgico de mi vida en Barcelona. Con Lepprince la conversación era pausada e intimista, un intercambio sedante y no una pugna constructiva, Lepprince escuchaba y entendía y yo apreciaba esa cualidad por encima de todo ${ }^{6}$.

Là encore, la prise de distance vis-à-vis des objets de passion accompagne l'avènement d'une ère nouvelle, même si la nostalgie des états tensifs passés se fait encore jour (on ne peut d'ailleurs avoir de nostalgie que pour ce qui n'est plus...).

6 E. MENDOZA, La verdad sobre el caso Savolta, Barcelona, Biblioteca de bolsillo, 1989, p. 93-94. [souligné par nous]. "Nos conversations ne se transformèrent pas en polémiques passionnées, comme cela s'était produit avec Pajarito de Soto peu de temps après notre rencontre : ces discussions enflammées qui maintenant, dans mon souvenir, prennent une grande importance et deviennent le symbole nostalgique de ma vie à Barcelone. Avec Lepprince, la conversation était pausée et intimiste, un échange sédatif et non pas un combat constructif ; Lepprince écoutait et comprenait et j'appréciais cette qualité par-dessus tout. » 
MUÑOZ MOLINA, dans son roman Beatus Ille, revient sur un épisode dramatique de la guerre civile espagnole, mais ce livre n'a pas les arêtes tranchantes de la rigueur de la science historique, pas plus qu'il n'est un roman historique venant distribuer un jugement ou un sens définitif sur les actions humaines. La fin du parcours de reconstruction réalisé par l'investigateur Minaya ressemble bien plus à une leçon de relativisme - également adressée au lecteur - ; la voix narrative du protagoniste Solana présente l'ensemble sous l'aimable tonalité qui réunit les complices d'un jeu :

Yo he inventado el juego, pero usted ha sido mi cómplice. Era usted quien exigía un crimen que se pareciera a los de la literatura y un escritor desconocido o injustamente olvidado que tuviera el prestigio de la persecución política y de la obra memorable y maldita, condenada, dispersa, exhumada por usted al cabo de veinte años. [...] Contruyámosle el laberinto que desea, pensé, démosle no la verdad, sino aquello que él supone que sucedió y los pasos que lo lleven a encontrar la novela y descubrir el crimen. [...] Y acaso la historia que usted ha encontrado sólo es una entre las varias posibles?

En fait, il semble bien que la tonalité du discours littéraire ait dû s'adapter aux changements intervenus dans le maniement des valeurs par les sociétés modernes. Le contrat moral fondé sur des normes non négociables, universelles, produit volontiers un contrat sémiotique de type polémique ${ }^{8}$ où dominent les notions de combat (contre le Mal, l'Ennemi, l'Erreur). Bien souvent, le discours des lois universelles

7 A. MUÑOZ MOLINA, Beatus Ille, Barcelona, Seix Barral, 1994 (1ère éd. 1985), p. 276-277. "Moi, j'ai inventé le jeu, mais vous avez été mon complice. C'est vous qui exigiez un crime qui ressemble à ceux de la littérature et un écrivain inconnu ou injustement oublié qui ait le prestige de la persécution et de l'œuvre mémorable et maudite, condamnée, dispersée, exhumée par vous au bout de vingt ans. [...] Contruisons-lui le labyrinthe qu'il désire, pensai-je, donnons-lui non pas la vérité, mais ce qu'il suppose s'être passé et les éléments qui l'amèneront à trouver le roman et à découvrir le crime. [...] Et peutêtre que l'histoire que vous avez trouvée n'en représente qu'une parmi plusieurs autres possibles. "

8 Contrat sémiotique polémique: Toute appropriation d'un objet par un sujet présuppose la perte de ce même objet par un autre sujet. 
s'appuie sur un style sémiotique tendu, impulsif, inquiet : celui de la littérature engagée, par exemple. L'avènement d'une culture de la responsabilité éthique a provoqué un déplacement vers un contrat sémiotique transactionnel ${ }^{9}$ : ce dernier est fondé sur l'échange, la conversation, le postulat de concorde et de compréhension mutuelle. Il ne s'agit plus de "contraindre" [cum stringere : serrer avec] ou de "convaincre » par la Loi ou la force, mais de persuader par l'accord et la négociation. L'impératif catégorique de Kant s'est vu (avantageusement ?) substitué par la morale communicationnelle d'Habermas. C'est cette mutation - au moins formelle - qu'incarne la détension sémiotique et son corrélât de changement de Voix discursive.

Après cette rapide incursion au sein de la catégorie sémiotique du Tensif, j'abandonnerai le domaine littéraire pour aborder quelques phénomènes appartenant à l'usage non poétique (au sens de Jackobson) du langage.

\section{STRATÉGIES DE PERSUASION : LA MÉTHODE DOUCE}

\section{L'effacement des opérations argumentatives}

Parler ici d'effacement ne revient pas à dire que les opérations argumentatives disparaissent en tant que telles, que le discours perd toute logique. Plus exactement, il s'agit d'un phénomène de diminution sensible de la présence des ces «mots du discours" chers à Oswald DUCROT, les connecteurs et opérateurs argumentatifs qui viennent spécifier explicitement les opérations à effectuer sur les énoncés mis en présence. Chaque connecteur, par exemple, est porteur d'une série d'instructions pragmatiques qui viennent contraindre les rapports à établir entre les propositions.

Marie-Pierre LAVAUD s'est livrée à ce propos à une fort intéressante comparaison entre des éditoriaux de journaux de

9 Contrat sémiotique transactionnel: L'appropriation d'un objet par un sujet ne présuppose pas la perte par un autre sujet. (Par exemple, si quelqu'un apprend quelque chose, il ne dépossède personne de cette information) 
la fin du XIXe siècle et de la fin du XXe siècle ${ }^{10}$. Appuyée sur l'analyse automatique du discours, la discrimination entre les deux périodes ne laisse aucune place au doute : de manière écrasante, le matériel argumentatif est sur-représenté dans les éditoriaux de la fin du XIXe, alors qu'il est sous-représenté dans ceux de la fin du XXe. La période actuelle se caractériserait donc par une moindre spécification des rapports logiques, par une implicitation de ces mêmes rapports, comme si le locuteur répugnait à porter la contrainte logique sur le devant de la scène discursive par l'emploi de morphèmes spécifiques. A ces éléments strictement pragmatiques font écho d'autres indices. L'emploi de la première personne, l'abondance des déictiques qui rapportent le propos à l'instance d'énonciation, la désignation explicite de l'interlocuteur/lecteur sont le propre des éditoriaux de la fin du XIXe, lesquels s'opposent à une époque présente qui répugne à convoquer explicitement un JE ou un TU; tout au contraire, on observe un comportement délocutif qui évite de convoquer la source et la cible des actes de parole. Ces "petits détails " contribuent à façonner deux Voix locutrices sensiblement distinctes : si l'éditorialiste ancien s'affichait ouvertement, prétendait conduire fermement son propos et guider avec la même énergie son lecteur, le nouveau s'efface derrière son propos, laisse les faits se présenter seuls et l'allocutaire calculer les inférences logiques sur la base du contenu sémantique des énoncés. Cette stratégie énonciative spécifique génère une Voix discursive différente : la force de la démonstration ${ }^{11}$ issue d'un face à face explicitement contrai-

10 M.-P. LAVAUD, «Fins de siècle et argumentation : les éditoriaux de Heraldo de Madrid et El País", in Notre fin de siècle. Culture hispanique, Dijon, Université de Bourgogne, Hispanística XX, Centre d'Etudes et de Recherches du XX ${ }^{\mathrm{e}}$ siècle, 1996, p. 143-163.

11 Le phonéticien Ivan Fónagy a réalisé une expérience qui corrobore l'observation faite dans le changement de ton des éditoriaux. Il a fait écouter plusieurs conférences prononcées dans des langues étrangères inconnues du public (français) et demandait ensuite aux auditeurs de tenter d'imaginer quelques traits du contenu, y compris psychologiques, des interventions. Les discours à caractère argumentatif et démonstratif prononcé étaient immanquablement pris comme signes d'un état d'esprit violent, ou en colère, de l'orateur. Comme quoi le discours que cherche explicitement à "con-vaincre » 
gnant (fin XIXe) a été substituée par le ton de la causerie fondée sur la connivence (fin XXe). Le discours reproduit dans ses structures un éthos de communication télévisuelle : ne surtout pas gesticuler, donner une impression de sérénité, incarner la sophrosunè ${ }^{12}$ des Anciens. L'effacement discursif des connecteurs et opérateurs logiques représente ainsi un symptôme au sein d'un faisceau de traits organisés en vue de « pauser le geste vocal ». Et comme nous allons le voir, cette tendance profonde est actualisée sur d'autres registres.

\section{La culture des euphémismes ${ }^{13}$}

Les organismes politiques, administratifs ou commerciaux, les médias, s'inscrivent, par l'emploi fréquent de l'euphémisme, dans un mouvement global que les publicitaires ont résumé sous le nom de «mieux-disant "-version communicante du «politically correct». Dans une société soft, il faut dédramatiser, limer les aspérités, éviter les sujets qui fâchent, ménager la face de l'autre, rendre à tout et à tous une dignité, en un mot $P O-S I-T I-V E R$, comme on l'a si bien compris chez Carrefour. Et si tout bambin convenablement éduqué apprend dès son plus jeune âge que l'on ne dit pas $j e$ veux, mais je voudrais ou j'aurais voulu, il ne sait pas encore qu'il est inconvenant d'appeler un chat un chat; il ignore en effet que les balayeurs sont des techniciens de surface, les régions pauvres des zones économiquement déprimées, ou que les arracheurs de sac de vieilles dames et autres incendiaires d'automobiles ne sont que de petits sauvageons, au fond bien sympathiques. La tendance euphémique du discours se présente comme le corrélat langagier de canons culturels et mentaux modernes caractérisés par la prolifération

avoue son caractère de violence contraignante déjà sur le plan phonétique et mélodique. Cf. I. FóNAGY, La vive voix. Essais de psycho-phonétique, Paris Payot, 1991.

12 Sophrosunè : tempérance de l'homme mûr, qui s'oppose à l'hybris juvénile.

13 Pour une étude plus exhaustive de cette question, on pourra se reporter à C. BOIX, "Ce que mieux parler veut dire. Les euphémismes », in Actes du Colloque International "Les débuts de siècle ", Dijon, UFR Langues \& Communication, Université de Bourgogne, 2000 . 
des «méthodes douces", des «versions soft " et autres «produits light».

Cela dit, l'étymologie du terme euphémisme - issu du grec - rappelle son origine lointaine : euphémisme vient d'euphêmismos, lui même issu de euphêmos: "de bon augure ". Le mot est composé du radical phême : "parole» et du préfixe $e u$ : «bien ". Parler par euphémismes revient en quelque sorte à prodiguer de «bonnes paroles", qui renvoient à des réalités placées sous les meilleurs auspices. C'est ainsi, par exemple, que les chômeurs ont retrouvé - à défaut de mieux - une activité sociale en devenant des demandeurs d'emploi. La pensée mythique, de tout temps, a tenté de conjurer les menaces du sort en ne mentionnant pas explicitement les réalités qui fâchent... les dieux. Ce mécanisme de base semble faire retour dans notre vie quotidienne, à cette différence près qu'il ne s'agit plus d'éviter d'encourir les foudres de l'Olympe, mais plutôt celles du commun des mortels : un plan de restructuration d'entreprise permettra au moins un temps d'expectative, une latence protestataire que n'aurait pas suscité un programme de mise à pied de 25\% du personnel; le Revenu Minimum d'Insertion (RMI) est autrement plus prometteur que les dénominations crues de Minimum Vital ou d'Allocation de Subsistance d'antan.

Ce qui se met en place sous les mots "soft ", c'est une chaîne de désignations contraires aux argumentations que pourraient développer les esprits chagrins. Face à l'entassement, la promiscuité, la violence présente dans les transports en commun, nous avons trouvé une réponse prometteuse en créant le nouveau métier "d'agent d'ambiance et d'espace » : voici des agents qui apportent déjà par leur nom «l'espace et l'ambiance», la sérénité des grands horizons. Plus il y a d'espace et plus il y a d'ambiance, plus on est heureux. Les euphémismes convoquent des topoï ${ }^{14}$ qui permettent d'argumenter dans le sens du bien, du beau et du progrès. Même l'égalité sociale y gagne, puisque les "promoteurs de vente" (anciens voyageurs de commerce) tutoient désignativement les "promoteurs immobiliers»; les

14 Nous prenons ici le terme Topö̈ au sens que lui donne Oswald DUCROT : orientation argumentative et axiologique présente dès le contenu lexical du mot. 
"techniciens de surface» partagent un bout de titre avec les " techniciens supérieurs "; les «administrateurs de propriétés urbaines $^{15}$ " sont quasiment des «administrateurs de biens». Dans le domaine du travail, rien n'échappe à cette orientation argumentative, souvent souterraine, qui fonctionne par rapprochements, par intégration dans des paradigmes axiologiques. Prenons un exemple courant : celui des «petits boulots". Un "petit boulot», ce n'est certes pas grandchose, si l'on ne considère le quantificateur "petit » que sous l'aspect lexical. Mais envisageons sa valeur du point de vue argumentatif et axiologique : les «p'tits boulots », les «p'tits papiers ", les «p'tits gars ", les «p'tits pères" comme les "petits enfants", les «sauvageons" ou les «petits malins", c'est affectueux, on les aime, car tout ce qui est petit est mignon: small is beautiful, comme disent nos voisins d'outre-Manche. Et l'essentiel est bien là : L'important, c'est l'appréciation axiologique qui vertèbre l'apparente fonction descriptive des mots et des désignations. Les euphémismes jouent sur les principes évaluatifs - lesquels constituent le niveau profond de la signification - pour inverser le sens de l'évaluation axiologique. Par exemple en substituant l'absence par une présence comme avec les «mallogés », les «mal-nourris », les "mal-aimés ». Restent à inventer les «mal-comprenants » pour désigner les imbéciles, et la liste sera complète... Ce principe générateur positivant se retrouve dans des expressions lexicales diverses et variées. Par exemple, porter cet artefact que l'on nomme une "perruque » risquait de suggérer la fâcheuse absence qu'il vient dissimuler... Qu'à cela ne tienne : les magasins spécialisés dans la vente de ce type d'accessoire parlent de " complément capillaire ». Si vous "complémentez », c'est que vous ne partez pas de rien! Le nombre des exemples que l'on pourrait adjoindre est quasiment infini, touche à tous les domaines dits "sensibles ": il suffit de penser aux déjà pudiques «erreurs de tir» des forces armées, transformées en "dommages collatéraux» sur Belgrade (Dommage pour

15 Traduction littérale de la désignation espagnole : «administrador de finca urbana" (concierges d'immeubles chics, regroupés derrière de hautes grilles...). Les promoteurs français ont trouvé l'équivalent : «Gardien-Régisseur». 
l'Ambassade de Chine, mais ce n'est qu'un effet malheureux parallèle à la réalisation d'un objectif positif). L'essentiel, comme nous l'avons répété à plusieurs reprises, c'est la dynamique profonde du renversement axiologique que porte en soi la désignation euphémique.

La Voix, la corporéité de la Loquence, ne peut que s'en trouver changée, également inversée : que l'on imagine par exemple toute la distance que suggère l'opposition entre les deux formulations suivantes d'un même acte de parole de refus :

/Il n'est en pas question/ vs

/Je crois que ça va pas être possible/

L'adoucissement de la Voix provoqué par la phraséologie «soft »-de même que son caractère trop poli pour être honnête - est à ce point devenu marquant et généralisé qu'il est épinglé par la chanson contemporaine ${ }^{16}$.

\section{De la propagande à la publicité}

A ses débuts, la promotion des produits de consommation était couramment nommée propagande. Les dictionnaires relèvent d'ailleurs toujours cette acception du mot ${ }^{17}$. Mais cette valeur est aujourd'hui tombée en désuétude et le terme consacré est celui de publicité. Un bref détour par les contenus et les usages de ces termes permet de voir, là encore, une logique de la détension, du changement du rapport à l'autre, une volonté de remplacer un mouvement explicite de persuasion par des formes qui se veulent littéralement inoffensives.

A l'origine, le terme latin PRO PA(N)GO possède une référence concrète issue de la culture agricole. Il s'agit de

16 «Je crois que ça va pas être possible» est en effet le titre d'une chanson - contemporaine et emblématique - du groupe toulousain Zebda.

17 La trace de cette valeur, temporellement fugace, apparaît aussi bien dans les dictionnaires français - où l'on conserve, sous l'entrée "publicité ", une rubrique généralement non commentée : propagande et publicité - que dans les dictionnaires espagnols : cf. Real Academia : PROPAGANDA 3. Acción o efecto de dar a conocer una cosa con el fin de atraer adeptos o compradores. 
l'idée d'une plantation (PANGO) que l'on étend par bouturage, qui ainsi croît et se multiplie «à travers » (PRO), occupe de plus en plus d'espace. De cette racine, la langue latine avait déjà tiré des sèmes plus génériques. C'est ainsi que le PROPAGATOR, qui signifiait le conquérant, assurait l'extension de son territoire d'origine et par là même la pérennité de son rayonnement. Ce n'est que plus tard que les valeurs notionnelles prendront le relief que nous leur connaissons, sans toutefois s'écarter de cette notion d'extension et de conquête. En effet, la propagande (littéralement $=$ ce qui doit être propagé) paraît inséparable d'une certaine dose de compétition : il faut gagner, conquérir du terrain sur quelque autre chose (la friche, les autres idées, l'ennemi, etc.). Sur le plan religieux, par exemple, on trouve dès 1622 la Congrégation de la Propagande (CONGREGATIO DE PROPAGANDA FIDE), organisée par Grégoire XV, dont la fonction était de répandre la foi et la religion catholique. La propagande renferme donc dans ses sèmes profonds l'image d'opération de conquête des esprits, un faire-croire expansionniste : Il faut d'ailleurs remarquer que l'expérience ultime faite de la propagande (transférée sur le plan politique) fut intimement liée à la guerre et aux conflits radicaux.

A la différence de la propagande, qui exprimait par sa nature originelle verbale l'idée d'une action, la publicité est un vocable issu de l'adjectif latin PUBLICUS. La publicité est donc la qualité de ce qui est public, connu du plus grand nombre : la racine adjectivale renvoie au résultat et moins à l'action qui permet de l'obtenir. Le sens dérivé de porter à la connaissance des gens, de la masse, du plus grand nombre, pointe vers un «simple " faire-savoir, beaucoup plus neutre que le faire-croire. Il ne s'agit plus d'une action - avouée comme intentionnelle - sur le récepteur, mais d'une simple monstration de ce qui n'était pas donné à voir antérieurement. A l'image du circuit commercial qui met en circulation un nouveau produit, objet de consommation, la publicité fait mine de se contenter de mettre en circulation un savoir, objet de communication. Instrument visant un public de masse, faire persuasif « soft », le message (massage ?) publicitaire joue sur l'ambiguïté d'une expression apparemment immédiate, ce qui ne l'empêche nullement d'ailleurs d'être une représentation 
parfaitement maîtrisée... Toute la différence est dans la manière : comme dans les éditoriaux de journaux, on se livre à des opérations de persuasion en ne déclarant surtout pas l'acte pragmatique visé par le message. Nous assistons à l'avènement d'une procédure fonctionnant par flashes de publicité, jouant sur l'immersion dans un univers qui constitue par touches un public en unité organique. Au sein de ces groupes, la communauté devient une sorte de ruche bourdonnante où chacun acquiert le sentiment de résonner à la vibration de l'autre. Tout se passe comme si la publicité donnait à ce même groupe le sentiment rassurant d'appartenance à une communauté homogène sublimée et apaisée dans la réduction de ses différences. Une Voix biaisée et holistique $^{18}$ parle avec douceur, sur le mode de la devinette ou de l'humour détendu, à des interlocuteurs en attente de cocooning ou de sur-protection maternelle.

\section{POUR NE PAS CONCLURE}

Les rapides exemples que j'ai choisis, volontairement hétérogènes sur le plan des types discursifs, pointent vers l'émergence d'une Voix particulière, vers une loquence de la dé-tension. Cette tendance nouvelle a sans doute beaucoup à voir avec la philosophie générale de la communication, dans une ère qui porte son nom. Le philosophe Habermas affirme que le langage lui-même renferme les instruments d'une «Morale communicationnelle»: toute négociation suppose un horizon commun, des règles du jeu partagées, des garants qui fondent la possibilité d'un échange et d'un accord. A côté de l'agir stratégique ou instrumental, organisé selon le système fins-moyens, il existe un agir communicationnel dirigé, par sa nature même, vers l'entente et l'intercompréhension. L'émergence insistante d'une Voix «soft» est-elle le signe profond de l'émergence d'une civilisation fondée sur la recherche de l'entente et de l'intercompréhension?

Cette forme de Loquence est-elle le moule dans lequel prend corps une éthique différente? Ou bien sommes-nous entrés dans un jeu virtuel plus sophistiqué qui ne représen-

18 Holistique : qui procède par retours constants pour édifier progressivement une structure de plus en plus complexe et efficiente. 
terait qu'une subtile manière d'affiner les stratégies de persuasion pour les adapter aux circonstances tout en leur conservant leur efficacité maximale ? Charles BALLY remarquait, il y a déjà fort longtemps, que l'atténuation relevait de la contrainte sociale :

[...]l'adaptation au milieu s'accompagne presque toujours d'une atténuation de la pensée et de l'expres$\operatorname{sion}^{19}$.

Tout comme les règles de conduite deviennent plus strictes à mesure que croît le flot de la circulation, l'apparition d'une communication toujours plus dense pourrait rendre nécessaire cette adaptation de la phraséologie aux autoroutes encombrées de l'information et de l'échange verbal. Que cette nouvelle Voix soit une "voix contrefaite" ou non, sa présence dominante est bien réelle dans le monde contemporain, telle une musique plus douce destinée à adoucir des mœurs souvent récalcitrantes...

19 Ibid., p. 297. [souligné par nous] 